\title{
Game-Theoretic Analysis of Renouncing Membership of a Party to Announce Candidacy
}

\author{
Jue-Shyan Wang ${ }^{1}$, Mei-Yin Lin ${ }^{2}$ \\ ${ }^{1}$ Department of Public Finance, National Chengchi University, Taipei, Chinese Taipei \\ ${ }^{2}$ Department of Economics, Shih Hsin University, Taipei, Chinese Taipei \\ Email: jswang@nccu.edu.tw, mylin@cc.shu.edu.tw
}

Received June 20, 2012; revised July 20, 2012; accepted July 28, 2012

\begin{abstract}
A game model is established to analyze the interaction in strategy when there is a candidate who renounces membership of a party to run for a position. We use the Bayesian Nash equilibrium and sequential equilibrium to discuss two models in which the candidate without nominations moves first or the decision maker of the other party moves first respectively. The main finding is that the equilibrium strategy of the decision maker of the other party is identical when the opponent runs for a position, regardless of who moving first. However, the probability of the candidate without nomination to run for a position is larger when he moves first.
\end{abstract}

Keywords: Bayesian Nash Equilibrium; Sequential Equilibrium

\section{Introduction}

In Taiwan, there are two ways for anyone to be a candidate: to be nominated by a party or to run for a position independently. The nominee will have full support from the party in the election campaign. However, a member in the party without nomination should renounce membership to run for a position. There were some famous examples of violating the party rule to elect independently in the electoral history of Taiwan. The examples were Lin Yang-kang in the presidential election in 1996, James Soong in the presidential election in 2000. There was an example in United States too. In spite of being a Democrat originally, George Wallace ran for the US president in 1968 on the American Independent Party.

In general, a candidate that doesn't acquire a party nomination has paid the penalty of breaking off relation with the original party. There may be some benefit for a member to renounce membership to run for a position. This paper intends to study the behavior of the candidate who withdraws from a party.

Only few economic studies have so far been made at this issue. Epstein (1967) demonstrates that the candidates nominated by the party can form the image in the voters' minds and this is important information for voting decision. Gallaher (1988) suggests that the party will control the members of congress by the power of nominations. Ware (1988) points out the leader of the party will be short of ability to carry out the nominations if there is a dispute inside the party.
The previous literature mostly focuses on the nominating process or the possible influence of nomination on the election or voter's decision. This paper sets up a game model to analyze the interaction in strategy when there is a candidate who renounces membership to run for a position. We concern with the behaviors of the candidate without nomination and the decision maker of the other party. We first consider the case of the candidate without nomination moving first. Bayesian Nash equilibrium is used to analyze this case where there is incomplete information. Secondly, we discuss the case of the decision maker of the other party moving first. We use sequential equilibrium to describe the equilibrium in the case where the information is imperfect.

\section{Model 1: The Candidate without Nomination Moving First}

Suppose the candidate who is not nominated by the party (denoted by player 1) makes his decision about whether to run for a position in the first. Then the player 2, denoting the decision maker of the other party, decides whether to campaign actively or inactively. Assume there are two types of player 2 . The first type of player 2 is denoted by type-s which occurs with probability of $q(q \in(0,1))$. Type-s player 2 thinks he will get benefit from the damaging image of the split in the opposite party even though he campaigns inactively and player 1 wins the election. This benefit of type-s player 2 in this state is set to be $f(f \in(0,1))$. Type- $w$ is defined as the second type of 
player 2 with probability of $1-q$. Type- $w$ player 2 thinks he will get the benefit of value $d$ if he participates in the election actively, no matter who the winner is in the election. The player 1 knows that the value of the probability is $q$, but doesn't observe in advance which type of player 2 he is dealing with.

Suppose the total vote these two players could get is fixed. And we assume the sum of the winning probability for the two players is fixed to be $r$. Then when both of the two players participate in the election actively, the winning probability of player 1 and player 2 are $r-e$ and $e$ respectively. It should be noted that we suppose the candidate renouncing the membership doesn't split any vote from his original party. Finally, the cost of electoral actions for player 1 is assumed to be the value of $a$. The cost of electoral actions for player 2 is set to be the value of $b$ when he campaigns actively. However, when player 2 campaigns inactively, there is no cost of electoral actions. We assume $r>a$ which implies that there really exists the incentive for player 1 to renounce the membership to run for a position. The whole structure of the game we described above is shown in Figure 1. And the normal form of the game is presented in Figure 2.

The symbol "A" indicates the player campaigns actively and the symbol "I" means the player campaigns inactively. We describe the strategy for player 2 with type- $s$ in the former and then the strategy for player 2 with type- $w$ in the latter. So the four pure strategies for player 2 are (AA, AI, IA, II). Let $A_{i}$ and $B_{i}$ denote the payoffs of player 1 and player 2 in state $i$ respectively, where $i$ belongs to the set of four strategies of player 2. The definitions for $A_{i}$ and $B_{i}$ are given in the following:

$$
\begin{aligned}
& A_{1} \equiv r-a-e \\
& B_{1} \equiv e-b+(1-q) d \\
& A_{2} \equiv r-a-q e \\
& B_{2} \equiv q(e-b) \\
& A_{3} \equiv r-a-(1-q) e \\
& B_{3} \equiv q f r+(1-q)(e+d-b) \\
& A_{4} \equiv r-a \\
& B_{4} \equiv q f r
\end{aligned}
$$

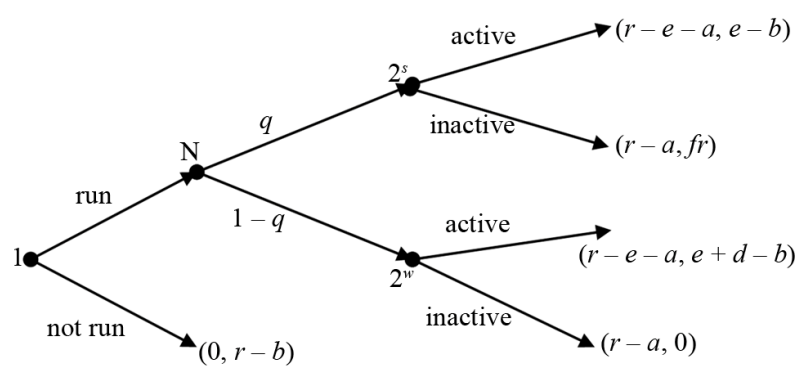

Figure 1. The extension form game of model 1.

\begin{tabular}{lccccc}
\hline & \multicolumn{4}{c}{ player 2} \\
\cline { 3 - 5 } Player 1 & run & AA & AI & IA & II \\
\cline { 3 - 5 } & not run & $A_{1}, B_{1}$ & (2) $A_{2}, B_{2}$ & (3) $A_{3}, B_{3}$ & (4) $A_{4}, B_{4}$ \\
& & $0, r-b(6) 0, r-b$ & (7) $0, r-b$ & (8) $0, r-b$ \\
\hline
\end{tabular}

Figure 2. The normal form game of model 1.

For the rationality of analysis, we assume $e-b>0$. This allows for the possibility of player 2 to campaign actively. Moreover, when the expected payoffs of running for a position and not running for a position are equal, player 1 will choose the strategy of not running.

Now we use the Bayesian Nash equilibrium to analyze this model. We first discuss the strategy of player 2 in equilibrium. By the assumption of $e-b>0$, the following two inequalities will be satisfied.

$$
\begin{aligned}
& e-b+(1-q) d>q(e-b) \\
& q f r+(1-q)(e+d-b)>q f r
\end{aligned}
$$

Therefore, if player 1 decides to run for a position, player 2 will not choose the strategies (AI) and (II). Comparing the expected payoffs of the strategies (AA) and (IA), we get the following result. When $e-b>f r$, player 2 will adopt the strategy (AA), otherwise he will select the strategy (IA). If the expected payoffs of these two strategies are equal, then he can choose either.

Since player 2 will not select the strategies (AI) and (II), we would compare the expected payoffs of player 1 among $A_{1}, A_{3}$ and 0 . There exist the following three situations:

1) $A_{1} \geq 0$

2) $A_{3} \geq 0>A_{1}$

3) $A_{3}<0$

Combining the player's principle for decision with the three situations stated above, the results of Bayesian Nash equilibriums could be summarized in Table 1.

From Table 1, we first conclude that when the expected payoff of player 1 is smaller $\left(A_{3}<0\right)$, player 1 is unwilling to run for a position because of the negative expected payoff. Secondly, type- $w$ player 2 is determined to campaign actively. However, type-s player 2 will campaign actively when player 1 runs for a position only if the payoff of player 2 is larger than that of campaigning inactively $(e-b>f r)$. The reverse is true. Finally, since $A_{3}$ is positively related to $q$, player 1 is very likely to run for a position when the proportion of type- $s$ in player 2 is large. The Bayesian Nash equilibriums in this model may be summarized in the following proposition.

Proposition 1. The decision maker of the other party with type- $w$ will certainly campaign actively. And type- $s$ player will campaign actively (inactively) if $e-b>(<) \mathrm{fr}$. Moreover, the higher the proportion $q$ is, the greater the probability of the candidate without nomination to run for a position is. 


\section{Model 2: The Decision Maker of the Other Party Moving First}

In model 2 , the decision maker of the other party decides whether to campaign actively first. And then the candidate without nomination makes the choice of whether to renounce membership to run for a position. Because of the reverse in the decision-making order, we exchange the notations of these two players in this model. That is, player 1 denotes the decision maker of the other party and player 2 denotes the candidate without nomination. The strategy and the corresponding payoff of each player are set to be the same with model 1 . Thus the extensive form of this game could be described as Figure 3.

Because there is uncertainty in the type of player who moves first, we use the concept of sequential equilibrium to analyze model 2. Define $p^{s}\left(p^{w}\right)$ as the probability of type-s (type- $w$ ) player 1 that campaigns actively. Let $t$ $(t \in[0,1])$ denote the probability for player 2 to run for a position. After observing player 1 campaigning actively, player 2 sets the belief that player 1 belongs to type- $s$ to be $\mu$. Then the expected payoff of player 2 when he runs for a position is:

$$
\mu(r-e-a)+(1-\mu)(r-e-a)=r-e-a
$$

Comparing the payoff shown in Equation (1) for player 2 with zero payoff of not running for a position, we suspect that player 2 will (not) run for a position when $r-e>(<) a$.

In the following, we discuss the behavior of player 1 . Given the probability that player 2 runs for a position is $t$, the expected payoff for type-s player 1 to campaign actively is:

$$
t(e-b)+(1-t)(r-b)=r-(r-e) t-b
$$

Comparing the payoff arranged in Equation (2) with the payoff of campaigning inactively $(f r)$, type-splayer 1 will campaign actively (inactively) when $t<(>)[(1-f) r-b] /(r-e)$.

The expected payoff of type- $w$ player 1 when he campaigns actively is:

$$
t(e+d-b)+(1-t)(r-b)=r-(r-e-d) t-b
$$

Comparing the payoff for player 2 presented in Equation (3) with the zero payoff of campaigning inactively, we conclude that type- $w$ player 1 is determined to campaign actively for the reason that $e-b>0$ and $r>e$. Summing up all the above arguments, the four sequential equilibriums are listed in Table 2.

Table 1. The Bayesian Nash equilibrium of model 1.

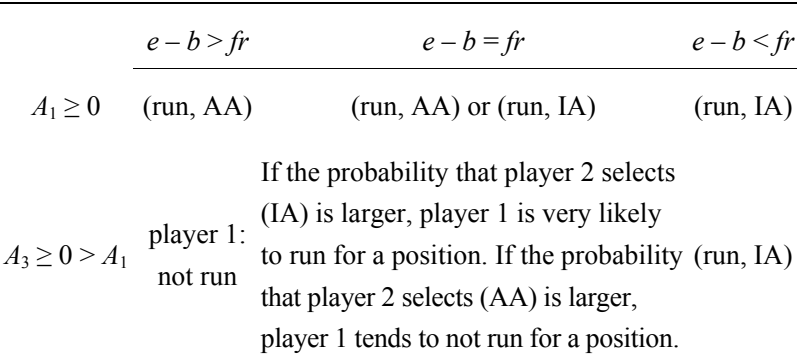

$A_{3}<0 \quad$ player 1: not run

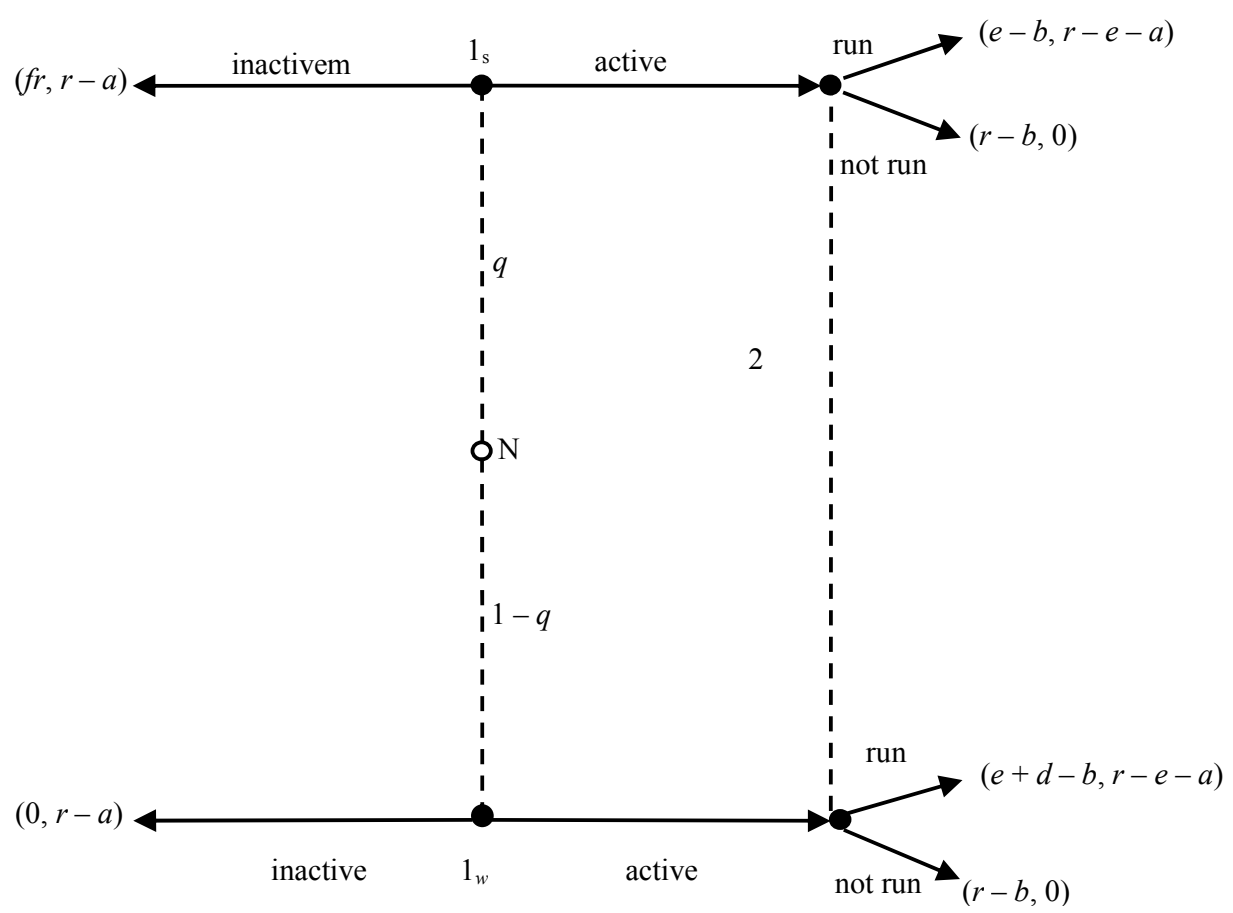

Figure 3. The extension form game of model 2. 
Table 2. The sequential equilibrium of model 2.

\begin{tabular}{cccc}
\hline & $\begin{array}{c}\text { Strategy of player 2 } \\
\text { (when player 1 } \\
\text { Strategy of player 1 } \\
\text { campaigns actively) }\end{array}$ & Belief & $\begin{array}{l}\text { Required } \\
\text { condition }\end{array}$ \\
\hline $\begin{array}{c}\text { (1) type- } s \text { : active } \\
\text { type- } w \text { : active }\end{array}$ & run & $\mu=q$ & $\begin{array}{l}r-e>a \\
e-b \geq f r\end{array}$ \\
(2) type- $s$ : inactive & run & & $r-e>a$ \\
type- $w$ : active & & $\mu=0$ & $e-b<f r$ \\
(3) type- $s$ : active & not run & $\mu=q$ & $r-e \leq a$ \\
type- $w$ : active & & & $r-b \geq f r$ \\
(4) type- $s$ : inactive & not run & $\mu=0$ & $r-e \leq a$ \\
type- $w$ : active & & & $r-b<f r$ \\
\hline
\end{tabular}

As Table 2 indicates, the required conditions for these four equilibriums are unrelated to the proportion of each type in player 1, but have relationships with the payoff of each player. We take the case of player 2 for example. If the left vote, which has subtracted the amount player 1 receiving from the total vote the two players getting, is larger (smaller) than cost of electoral actions, player 2 will (not) run for a position (i.e. $r-e>(<) a$ ). In addition, the decision of player 1 is more complicated. Type- $w$ player 1 will campaign actively without doubt. For type- $s$ player 1 , considering the state that the opponent runs for a position, if the expected payoff of competing $(e-b)$ is larger (smaller) than that of not competing $(f r)$, type-s player 1 will campaign actively (inactively). Moreover, knowing that the opponent will not run for a position if player 1 campaigns actively, type-s player 1 will really campaign actively when expected payoff of competing alone $(r-b)$ is larger than that of leaving the opponent competing alone $(f r)$. This leads to the following proposition.

Proposition 2. The sequential equilibrium in this model have no connection with the proportion of each type in the other party. The candidate without nomination will (not) run for a position if $r-e>(<) a$. The decision maker of the other party with type- $w$ will campaign actively certainly. Type- $S$ player will campaign actively (inactively) if $e-b>(<) f r$ when the opponent runs for a position. And Type- $S$ player will campaign actively (inactively) if $r-b>(<) f r$ when the opponent doesn't run for a position.

Now we compare the equilibriums in these two models. For the decision maker of the other party, the equilibrium strategies are identical in these two models when the opponent runs for a position. Namely, type- $w$ player will campaign actively certainly. The decision of type-s player is based on the comparison between the values of $e-b$ and $f r$ when the opponent runs for a position, and the values of $r-b$ and $f r$ when the opponent doesn't run for a position. Consider the equilibrium strategy of the candidate without nomination. He decides to run for a posi- tion if $r-a-(1-q) e>0$ when he moves first, and if $r-a-e>0$ when he moves late. Since $1-q>0$, candidate without nomination is more likely to run for a position when he moves first. This draws the following proposition.

Proposition 3. The probability of the candidate without nomination to run for a position is larger when he moves first. The decision strategy of the decision maker of the other party is identical when the opponent is running for a position, regardless of who moving first.

Proposition 3 may result from the fact that the first moving candidate without nomination has incomplete information of the other party. Thus his decision is based on the prior belief in the distribution of the opponent's type and it leads to him facing more risk.

Because of the property of proposition 3 , the decision maker of the other party with type-s hopes to stand in the state of model 1 when he tends to campaign inactively. In other words, the decision maker of the other party with type- $s$ wants the opponent to move first to raise the probability of the opponent to run for a position. In reality, the decision maker of the other party can delay the nominating date (campaigning inactively) to stimulate the opponent to run for a position. On the contrary, the type- $s$ player hopes to be in the state of model 2 when he prefers to campaign actively. It will decrease the probability of the opponent to run for a position. That is, the decision maker of other party will finish the nominating process as soon as possible (campaigning actively) which reduces the opponent's intention of running for a position. Thus we may go on from this to the proposition 4.

Proposition 4. If $e-b<(\geq) f r$, the decision maker of the other party with type-s prefers to move late (first).

\section{Conclusions}

This paper applies a game model to analyze the interacttion in strategy when there is a candidate who renounces membership of a party to announce running for a position. We discuss two models in which the candidate without nominations moves first and the decision maker of the other party moves first respectively. The main finding is that the equilibrium strategy of the decision maker of the other party is identical when the opponent is running for a position, regardless of who moving first. However, the probability of the candidate without nomination to run for a position is larger when he moves first. Therefore, the decision maker of the other party may prefer to move first if he wants to campaign actively.

In this paper, we set some simplified assumption which can be relaxed. First, the candidate without nomination may split the vote which belongs to the original party. Moreover, if the two players can negotiate in advance, the negotiation result may change the strategy of the player. 
However, this paper would be a good stepping-stone for the future study about this topic. And it provides the suggestion in strategy for the candidate or the party who would deal with such issue.

\section{REFERENCES}

[1] L. D. Epstein, "Political Parties in Western Democra- cies," Praeger, New York, 1967.

[2] M. Gallagher, "Conclusion," In: M. Gallagher and M. March, Eds., Candidate Selection in Comparative Perspective: The Secret Garden of Politics, SAGE Publications, London, 1988.

[3] A. Ware, "Citizens, Parties and the State," Princeton University Press, Princeton, 1988. 